\title{
Chapter 11 \\ Mental Health Care \\ for Survivors and Healthcare \\ Workers in the Aftermath \\ of an Outbreak
}

\section{Jacqueline Levin}

The approach to mental health care for survivors is informed by a number of individual, social, and cultural factors. One must first consider the psychiatric sequelae of surviving the illness, its complications, and the complications of its treatments. In the acute phase of illness, even small foci of infection can produce psychiatric symptoms ranging from mood changes and irritability to cognitive dysfunction to psychosis. Neuropsychiatric manifestations may even present as the first signs of infection in an otherwise well-appearing patient. Hematogenous spread of bacteria or virus to the central nervous system can produce meningitis associated with significant morbidity and mortality, presenting symptoms including headache, nausea, nuchal rigidity, confusion, lethargy, and apathy to be confirmed by the examination of CSF. Bacterial meningitis may also result in brain abscess, with seizures and various psychiatric symptoms prevailing depending on the size and location of the abscess. Successful treatment with empirical

\footnotetext{
J. Levin $(\bowtie)$

Department of Psychiatry, North Shore University Hospital, Manhasset, NY, USA
} 
antibiotics and primary excision of the abscess may still result in persistent psychiatric symptoms. In cases of viral encephalitis, psychiatric symptoms are very common in the acute phase and recovery, especially mood disorders. Major disability can result, including symptoms of depression, amnestic disorders, hypomania, irritability, and disinhibition (sexual, aggressive, and rageful) even months after recovery. Psychosis may also rarely result. Standard treatments with antidepressants, stimulants, mood stabilizers, neuroleptics, and electroconvulsive therapy should be applied [1].

Individuals may suffer potentially permanent cognitive deficits secondary to illness or its treatments that will require cognitive rehabilitation. In cases of delirium, if the resultant encephalopathy is severe or persistent, pharmacologic interventions with antipsychotics (such as haloperidol $0.5-20 \mathrm{mg}$ / day) and mood stabilizers (such as valproic acid up to $60 \mathrm{mg} / \mathrm{kg}$ / day) should be considered. In addition, psychosocial interventions will need to be implemented to maintain safety and care for someone who may no longer be able to care for themselves. Additional consideration on this topic is provided in the chapter entitled Neuropsychiatric Sequelae of Infectious Outbreaks.

In the wake of an infectious disease outbreak, the loss of functioning imparted by illness may leave survivors feeling demoralized, helpless, and in a state of mourning over the loss of the person they used to be. If the patient experiences marked distress or significant impairment in social or occupational functioning, they may meet DSM-V criteria for adjustment disorder. Therapeutic interventions in those instances should focus on helping individuals regain a sense of autonomy and mastery through rehabilitation. It is helpful to focus on gaining immediate control over some specific aspects of their lives, as well as helping the persons identify and link with agencies and supports in the community [2]. Psychotherapy, both individual and group therapy, if available, can help survivors come to terms with the loss of functioning.

If the patient is left with significant depressive symptoms meeting DSM-V criteria for major depressive disorder, the psychopharmacological approach may be warranted; selective serotonin reuptake inhibitors or serotonin-norepinephrine 
reuptake inhibitors should be considered in such cases. Concurrent insomnia may be treated with melatonin, trazodone, ramelteon, or any available sedatives-hypnotics. Prescribers should be aware of drug-drug interactions and cytochrome P450 interactions between selected psychotropics and medications prescribed by infectious disease physicians in treating survivors. Patients who are at increased risk of developing delirium (i.e., elderly, dementia, and brain disease) should also be monitored for changes in mental status, attention, alertness, and orientation. Psychotherapy (cognitive behavioral therapy, supportive psychotherapy, and psychodynamic psychotherapy) may also be of clinical benefit if available. Enlisting local cultural and spiritual leaders may also help build hope and confidence.

Another important consideration is that proximity to and survival from life-threatening events (in this case illness) are known risk factors for the development of trauma-based disorders, including acute stress disorder and posttraumatic stress disorder (PTSD). PTSD is characterized by intrusive thoughts, nightmares, and flashbacks of past traumatic events, avoidance of reminders of trauma, hypervigilance, and sleep disturbance leading to significant social, occupational, and interpersonal dysfunction. In the aftermath of pandemics, increased psychiatric screening and surveillance is recommended to address acute stress disorder, posttraumatic stress disorder, depressive disorders, and substance abuse.

In the short-term aftermath, psychological first aid can be administered to patients by public health and public behavioral health workers. Such interventions focus on establishing a respectful, supportive rapport, triaging critical needs, normalizing stress and grief reactions, supporting positive thoughts about the future, and teaching mindfulness-based techniques to decrease the levels of stress and hyperarousal (i.e., deep breathing, progressive muscle relaxation, and guided imagery). Normalizing angry feelings while decreasing anger-driven behaviors can also play a therapeutic role [2].

In the long-term aftermath of a pandemic, trauma-focused therapies and pharmacological treatments may be indicated. Once a diagnosis of PTSD is made, treatment should be 
initiated promptly. First-line treatment consists of traumafocused cognitive behavioral therapy (CBT) to help reduce pessimistic and catastrophic thoughts about the future. Exposure therapy and eye movement desensitization and reprocessing (EMDR) therapies may also be utilized. If these therapeutic modalities are not readily available, selective serotonin reuptake inhibitors (SSRIs) and serotonin-norepinephrine reuptake inhibitors (SNRIs) can also be considered first-line treatments, to be administered for a duration of at least 6-12 months to prevent recurrence and relapse. Monotherapy or adjunctive therapy with quetiapine may also be considered. Alpha-adrenergic receptor blockers such as prazosin could be used for sleep disruption and nightmares, either alone or in conjunction with an antidepressant [3].

Special consideration should also be given to individuals with preexisting mental health issues who may experience setbacks, relapses, and impairments in functioning. More vulnerable patients with serious and persistent mental disorders such as primary psychotic illnesses or developmental disorders are likely to experience destabilizing disruptions in routine and access to medications/treatments. Psychotic, manic, or depressive symptoms may be intensified due to stress; increasing standing psychotropic medications may be indicated. Preexisting anxiety and substance use disorders are likely to worsen in the face of constant fear and distress. It is helpful to provide patients with a supply of PRN or "asneeded" extra tablets of antipsychotics or benzodiazepines as the pandemic unfolds to treat worsening symptoms. It is also prudent to enlist these patients' families and social supports to warn them of the risk for psychiatric destabilization and provide them with specific examples of worsening psychiatric symptoms to be on the lookout for. A safety plan and communication strategy should be developed with the patient and his or her family in the aftermath of a pandemic, with attention paid to potential barriers imposed by the pandemic (i.e. pharmacy closures, difficulty accessing medications). When possible, it may be prudent to prescribe a few months' additional supply of medications to be entrusted to a reliable 
family member. Increased monitoring is prudent in the aftermath of a pandemic with bimonthly or even weekly visits, depending on the severity of illness. For patients who are unable to access their usual providers, telepsychiatry can be a helpful substitute where available. Mental health professionals should be trained in the assessment of suicidality and safety concerns which may arise in the setting of acute anxiety, disability, bereavement, and multiple losses.

As a special consideration, it is worth noting that survivors of pandemics may find themselves the targets of pronounced stigma and rejection by their local communities. Affected individuals may blame themselves, and they may be prevented from returning to their homes or workplaces [4]. Entire cultural groups, communities, and geographic populations may become targets of stigmatization, which may serve as a barrier to seeking care [5]. In these cases, validating the experience of the stigmatized person is of utmost importance. In some communities, survivors of pandemics have been lauded as heroes by nongovernmental agencies in an attempt to decrease stigma [4]. Fostering resilience in such persons and their communities can help them to reclaim a sense of self-efficacy and fortitude in the face of adversity [6].

Just as patients experience significant emotional impacts in the course of a pandemic, so too will the brave and selfless healthcare personnel who are charged with the responsibility of providing aid to the infected. Their burden, however, is compounded by their high and persistent risk for exposure and death, separation from their loved ones which may be either enforced or due to prolonged work shifts, seeing traumatic images of their disfigured or dying patients, working during surge conditions in overburdened settings with chronically scarce supplies and medications/vaccines, experiencing hopelessness due to massive human losses in spite of their best efforts to provide care, managing human remains, experiencing workforce quarantine, witnessing the death of their colleagues, lack of reinforcements and replacements, and their own fatigue and burnout, to name a few of the many traumas they must endure in the course of their service [7]. 
It, therefore, does not come as a surprise that studies of nurses who treated SARS patients during the 2003 outbreak indicated high levels of stress and $11 \%$ rates of traumatic stress reactions, including depression, anxiety, hostility, and somatization symptoms [8]. One study showed that even 1 year after the SARS outbreak in 2003, healthcare worker SARS survivors still had persistently higher levels of stress and psychological distress than non-healthcare worker SARS survivors [9]. Similar findings have been reported in multiple studies indicating acute and persistently elevated stress levels as well as other emotional sequelae of healthcare workers during and after pandemic disease outbreaks [10-12]. Those findings indicate that left unaddressed, emotional needs and wounds of healthcare personnel grappling with an outbreak can reverberate long, perhaps for many years, after an outbreak has abated.

Healthcare personnel working at great personal peril will, therefore, require frequent and clear communication regarding the status of the pandemic and developments as they unfold. Communication at every level should be monitored, with systems in place to bidirectionally transmit news among healthcare workers, their administration, healthcare facilities, and the government [10].

Leadership, structure, and clear delineation of duties and responsibilities are critical. Determining staffing needs and establishing predictable schedules will lay a stable foundation for healthcare workers and ground them in the face of other destabilizing forces. Healthcare workers on the frontlines should be supported to the fullest extent possible as the pandemic unfolds to prepare for what is to come. Educational materials should be developed and provided that can outline what healthcare workers might expect in the course of their duties, including common reactions and stressors they may encounter from the public, patients, their friends and families, or from within themselves. This is of utmost importance, as an unprepared workforce may feel afraid to serve; in a survey of over 6400 healthcare workers across 47 facilities in the New York metropolitan region, only $48.4 \%$ said they would 
be willing to report to work during an outbreak of SARS, most frequently citing fear for personal or family safety as the reason they were unwilling to work [13].

Given the real and understandable fear of contracting illness, comprehensive and repeated training on infection control and how to use personal protective equipment can help increase the confidence of the workforce that their personal safety will be maintained. Healthcare personnel should also be offered periodic health assessments to reassure them of their physical well-being [8]. Preparations should also center on immunization programs, available vaccines for frontline healthcare personnel, availability of prophylactic medications, and assurances that their concerns and needs will be heard and met [14].

A study of the psychological impact of the 2003 SARS outbreak on healthcare workers in Singapore found that support from supervisors and colleagues was a significant negative predictor for psychiatric symptoms and PTSD, in addition to clear communication of directives and precautionary measures which also helped reduce psychiatric symptoms [15]. Buddy systems pairing more and less experienced healthcare workers can help not only to transfer skills, but also to reduce social isolation and promote a sense of support and interconnectedness [10]. The experience of being a healthcare worker during a pandemic is both isolating and stigmatizing; having a partner to share the experience with would be beneficial on multiple levels.

Administrators can improve the situation by being attentive to the psychological, physical, spiritual, and psychosocial needs of healthcare workers. Systems should be implemented for rest and relief of duties to prevent burnout; it is also prudent to limit overtime [2]. Programs promoting well-being incorporating mindfulness and relaxation techniques can help healthcare workers develop self-help skills during times of increased stress; once learned, they may also be able to pass such skills on to their patients. Workforce resilience programs and self-care strategies should be promoted. Teamwork and morale-building activities should also be promoted, as 
well as wellness breaks. It may also be meaningful to plan staff-appreciation events and verbally acknowledge their ongoing efforts [2]. Spiritual leaders from the faith-based community may also be called upon to provide spiritual guidance to affected healthcare workers who would find tremendous comfort in such an outlet.

It is also important to remember that healthcare workers will have their own sick family members, childcare issues, and personal affairs impressing upon them from the outside world, which can leave them feeling pulled between a sense of duty to their patients and their loved ones. Psychosocial programs that are mindful of providing services for the families of healthcare workers can go a long way in supporting staff and protecting morale. Lending cellular phones, laptops, or tablets to healthcare workers and their families to ensure they are able to maintain ongoing communication, as well as providing updates on websites and hotlines, can also help healthcare workers feel they are still interconnected with their families and may alleviate some of the real pressures that are felt. Furthermore, healthcare workers should be regularly reminded and trained in infection control measures when they return home; for example, reminding staff of handwashing and to change clothes before entering their homes to protect family members. Providing disposable scrubs or garments especially for wear in the hospital may also help decrease healthcare workers' anxiety about transmitting illness to their families back home [2]. It may also help to designate healthcare workers a specialized status within the community, given the crucial public service role they play. For example, providing specialized identification cards that might prevent them from waiting in lines at gas stations or supermarkets, as well as fair compensation and a stipend for their families, may further promote a sense of professional pride and goodwill and may help counteract the negative impact of the stigma that they may endure.

Lastly, employee assistance programs should target healthcare personnel who have developed traumatic, affective, or anxiety disorders as well as those struggling with increased substance use disorders. Increased mental health monitoring 
is advised, given healthcare workers' proximity and repeated exposure to traumatic experiences, as well as the welldocumented evidence of the persistent distress they are likely to experience. They should be considered a high-risk group for developing psychopathology in the aftermath of a pandemic and they should be given the same consideration and nurturing of any other high-risk population identified. Healthcare workers should have ready access to psychiatric care, pharmacologic interventions, and both individual and group psychotherapy. They should be reassured that their families will receive the same.

Practitioners tasked with treating patients in the aftermath of a pandemic will face challenges in providing standard care, both due to infrastructural and crisis-related adversities, as well as secondary to unique biological changes imparted by the disease itself. It is important for practitioners to be aware of common drug interactions, dosing, and titration strategies, and special considerations for different classes of psychopharmacological agents used. This section aims to review and summarize pertinent aspects of psychopharmacological agents which may be of use to future practitioners who find themselves providing psychiatric care in the wake of a pandemic.

Antidepressants are first-line agents for a number of psychiatric conditions that may be encountered in the aftermath of a pandemic. Such diagnoses include mood disorder secondary to a general medical condition, major depressive disorder, posttraumatic stress disorder, dissociative disorder, obsessive-compulsive disorder, and generalized anxiety disorder, to name a few. To identify and treat major depressive disorder, the psychiatric interview should focus on the psychological symptoms of depression (i.e., sad mood, anhedonia, hopelessness, worthlessness, guilt, and suicidality) rather than the vegetative symptoms (i.e., sleep disturbance, appetite change, psychomotor changes, and decreased concentration and energy), which may be of lower yield in the setting of acute medical illness. Depression should also be distinguished from hypoactive delirium, which may also present 
with diminished appetite, sleep disturbance, and an appearance of apathy (in the case of delirium, treatment with antipsychotics will be more effective than addition of an antidepressant).

An adequate trial of an antidepressant is defined as 12 weeks of antidepressant therapy at an effective therapeutic dose. It is helpful to establish expectations with patients by reminding them that daily use is important (rather than asneeded use), that symptoms may take 2-4 weeks before they begin improving, and that common side effects such as nausea, diarrhea, headache, and sexual dysfunction may be expected. Patients age 24 and younger should be monitored for worsening suicidal ideation. For patients with significant concurrent anxiety, a slow titration may be most appropriate with temporary use of benzodiazepines until the antidepressant takes clinical effect (e.g., lorazepam $0.5-1 \mathrm{mg}$ orally two to three times per day). If the drug is not working within 6-8 weeks, the patient may require a dose increase or a switch should be considered. Providers should treat until remission or a significant reduction in symptoms is observed, continuing treatment for 1 year for the first episode of major depressive disorder and indefinitely if there have been two or more episodes.

There are six principal selective serotonin reuptake inhibitors in common use: fluoxetine, sertraline, citalopram, escitalopram, paroxetine, and fluvoxamine. The global accessibility of these agents may vary. Fluoxetine has a dose range from 10 to $80 \mathrm{mg}$ and has the longest half-life (2-3 days), which makes it an ideal choice for patients in whom there are concerns for compliance or consistent access to medication. Sertraline has a dose range of 25-200 mg, and its wide range of dosing making it a good choice for elderly patients or for those who may be sensitive to side effects. Fluoxetine and sertraline have no renal dose adjustment, but a lower or half dose is recommended for patients with hepatic impairment. Citalopram doses range from 10 to $40 \mathrm{mg}$, but should not exceed more than $20 \mathrm{mg} /$ day for patients over age 60 or if the hepatic impairment is present. There is no dose adjustment for mild/ moderate renal impairment, but caution should be used in 
severe impairment. It is important to note that citalopram should not be combined with other QTc prolonging agents (applies to antimicrobials such as erythromycin, clarithromycin, fluoroquinolones, antifungals, and antimalarials) for increased risk of torsades de pointes [16]. Escitalopram, an enantiomer of citalopram, has dose ranges from 5 to $20 \mathrm{mg}$, should not exceed more than $10 \mathrm{mg} /$ day in the elderly or in cases of hepatic impairment, or if severe renal impairment is present. Paroxetine doses range from 20 to $40 \mathrm{mg}$, with only $10 \mathrm{mg} /$ day recommended in cases of renal or hepatic impairment. It has the shortest half-life of all the SSRIs (21 hours), resulting in an uncomfortable discontinuation syndrome and may not be ideal for patients with interrupted access to care/ medications. Side effects of sedation, weight gain, constipation, and dry mouth may make it a favorable option, however, for specific patients. Lastly, fluvoxamine doses range from 100 to $200 \mathrm{mg}$; however, many drug-drug interactions are associated with its use and should be monitored for.

Clinically significant interactions exist between selective serotonin reuptake inhibitors and several antiretrovirals in the setting of HIV/AIDS. For example, SSRIs shown to have decreased metabolism in the setting of ritonavir include sertraline and citalopram, but alternatively, the levels of fluoxetine and fluvoxamine are both decreased by nevirapine. Fluoxetine and fluvoxamine can both increase the levels of amprenavir, delavirdine, efavirenz, indinavir, lopinavir/ritonavir, nelfinavir, ritonavir, and saquinavir [17].

Tricyclic antidepressants have common side effects such as drowsiness, confusion, dizziness, weight gain, hypotension, and tachycardia, as well as anticholinergic side effects including dry mouth, blurred vision, decreased gastrointestinal motility, and urinary retention. Some of these side effects can be taken advantage of in the setting of HIV/AIDS, specifically weight gain, increased sleep, and decreased diarrhea [17]. Mirtazapine 7.5-45 $\mathrm{mg}$ at bedtime similarly may be a good choice in patients with postinfectious cachexia and exhaustion as it promotes weight gain and can cause a significant sedation, making it suitable for patients suffering from 
insomnia. Bupropion 75-450 mg/day can be helpful in postinfectious anergia, but prescribers should bear in mind that it lowers the seizure threshold. Tricyclic antidepressants and serotonin-norepinephrine reuptake inhibitors are useful if there is also concurrent neuropathic pain or a lingering inflammatory process that persists following some viral infections; for example, amitriptyline $10-400 \mathrm{mg}$ at bedtime, duloxetine 60-120 mg/day, or venlafaxine 75-225 mg/day.

Antimicrobial drugs themselves have had prominent associations with delirium and a host of other psychiatric side effects. For example, antibacterials such as quinolones have been associated with psychosis, paranoia, mania, agitation, and Tourette-like syndrome, and procaine penicillin has been associated with delirium, psychosis, agitation, depersonalization, and hallucinations. Mefloquine and other antiparasitic/ antimalarial drugs have been associated with confusion, psychosis, mania, depression, aggression, anxiety, and delirium. Antituberculous drugs such as cycloserine have been associated with agitation, depression, psychosis, and anxiety. Antivirals such as amantadine have been associated with psychosis and delirium, and interferon treatment is frequently associated with depression [1].

In addition to being cognizant of the side effects of the treatments themselves, drug-drug interactions between antimicrobials and psychotropic drugs abound. Psychiatric care providers should exercise caution when utilizing specific psychotropics (i.e., antipsychotics or tricyclic antidepressants) in the setting of other QTc interval-prolonging agents such as erythromycin or ketoconazole, due to increased risk of ventricular arrhythmias and torsades de pointes. Providers should keep in mind that linezolid is an irreversible monoamine oxidase- $\mathrm{A}$ inhibitor and isoniazid is a weaker monoamine oxidase inhibitor - so the serotonin syndrome or hypertensive crisis can result if serotonergic antidepressants or other sympathomimetics (such as meperidine, which is an opioid analgesic) are coadministered. Antimalarials have been shown to increase the levels of phenothiazine neuroleptics. Clarithromycin and erythro- 
mycin can increase carbamazepine, buspirone, clozapine, alprazolam, and midazolam levels. Quinolones may increase clozapine and benzodiazepine levels but reduce benzodiazepine effect via the GABA receptor. Lastly, providers should be aware that isoniazid can increase haloperidol and carbamazepine levels [1].

Psychiatric care providers should be aware of the myriad complications of corticosteroid use, seen in up to $6 \%$ of patients presenting with significant neuropsychiatric manifestations. Anxiety, mania, delirium, or psychosis may present with the administration of corticosteroids, and a dosedependent relationship has been observed. In most cases, a reduction of corticosteroid dose will improve symptoms; however, if this strategy is not possible or ineffective, antipsychotics or mood stabilizers should be used [18]. In patients presenting with predominantly manic symptoms, special consideration should be given to medical comorbidities when selecting a mood stabilizer. Lithium may be difficult to administer in the setting of renal dysfunction, electrolyte abnormalities, or fluid shifts. Valproic acid may be relatively contraindicated in patients with significant liver disease or pancreatitis. Carbamazepine has antidiuretic actions, has quinidine-like effects on cardiac conduction, and has been associated with aplastic anemia and leukopenia which prescribers should bear in mind.

\section{Conclusion}

Providing psychiatric care to survivors and healthcare workers in the aftermath of a pandemic outbreak is a complicated, but crucial, imperative in the service of reducing the burden of human suffering. Challenges will abound on multiple levels, but there is no substitute for preparedness. Knowledge of assessment, differential diagnosis, medical complications, and treatment will aid the psychiatric care provider in developing a treatment approach for these patients who are most vulnerable during their greatest time of need. 


\section{References}

1. Levenson J. Chapter 27: Infectious diseases. In: Levenson J, editor. Textbook of psychosomatic medicine. 2nd ed. Washington, DC: American Psychiatric Publishing Press; 2010. p. 615-35.

2. Havice-Cover PJ, Drennen C. Pandemic influenza: quarantine, isolation and social distancing [Internet]. Colorado: The Colorado Department of Human Services Division of Mental Health. Available from: http://www.realisticpreparedness.com/ downloads/PanFluQuarantineIsolation.pdf

3. Stein MB. Pharmacotherapy for posttraumatic stress disorder in adults. Post TW, editor. UpToDate. Waltham: UpToDate Inc. http://www.uptodate.com. Accessed 16 Dec 2018.

4. Reardon S. Ebola's mental-health wounds linger in Africa. Nature. 2015;519:13-4.

5. Honermann B. An "epidemic within an outbreak:" the mental health consequences of infectious disease epidemics. 2015 Feb 26 [cited 2018 Dec 16]. In: O'Neill Institute for National \& Global Health Law Blog [Internet]. Washington: O'Neill Institute. [2007] -. [about 3 screens] Available from: http://oneill. law.georgetown.edu/epidemic-within-outbreak-mental-healthconsequences-infectious-disease-epidemics/

6. Missouri Department of Health and Senior Services. Pandemic influenza plan: psychosocial services preparedness [Internet]. 2018 September. [Cited 2019 Jan 11]. Available from: https:// health.mo.gov/emergencies/panflu/pdf/panfluplanpsychosocial. pdf

7. Shultz JM. Mental health consequences of infectious disease outbreaks [Webinar training course]. Institute for Disaster Mental Health at SUNY New Paltz and New York Learns Public Health. Presented 2017 Jan 27. [Cited 2019 Jan 11]. Available from:https://www.urmc.rochester.edu/MediaLibraries/ URMCMedia/flrtc/documents/Slides-MH-CONSEQUENCESOF-ID-OUTBREAKSV2.pdf

8. Chen CS, Wu HY, Yang P, Yen CF. Psychological distress of nurses in Taiwan who worked during the outbreak of SARS. Psychiatr Serv. 2005;56(1):76-9.

9. Lee AM, Wong JGWS, McAlonan GM, Cheung V, Cheung C, Sham PC, et al. Stress and psychological distress among SARS survivors 1 year after the outbreak. Can J Psychiatr. 2007;52:233-40. 
10. Styra R, Hawryluck L, Robinson S, Kasapinovic S, Fones C, Gold WL. Impact on health care workers employed in high-risk areas during the Toronto SARS outbreak. J Psychosom Res. 2008;64:177-83.

11. Chan AO, Huak CY. Psychological impact of the 2003 severe acute respiratory syndrome outbreak on health care workers in a medium size regional general hospital in Singapore. Occup Med (Lond). 2004;54:190-6. https://doi.org/10.1093/occmed/kqh027.

12. McAlonan GM, Lee AM, Cheung V, Cheung C, Tsang KW, Sham $\mathrm{PC}$, et al. Immediate and sustained psychological impact of an emerging infectious disease outbreak on health care workers. Can J Psychiatr. 2007;52(4):241-7.

13. Qureshi DK, Gershon MRRM, Sherman MMF, Straub MT, Gebbie ME, McCollum MM, et al. Health care workers' ability and willingness to report to duty during catastrophic disasters. $\mathbf{J}$ Urban Heal. 2005;82:378-88.

14. Levin PJ, Gebbie EN, Qureshi K. Can the health-care system meet the challenge of pandemic flu? Planning, ethical, and workforce considerations. Public Health Rep. 2007;122(5):573-8.

15. Chan AO, Huak CY. Psychological impact of the 2003 severe acute respiratory syndrome outbreak on health care workers in a medium size regional general hospital in Singapore. Occup Med (Lond). 2004;54:190-6. https://doi.org/10.1093/occmed/kqh027.

16. Yap YG, Camm AJ. Drug induced QT prolongation and torsades de pointes. Heart. 2003;89(11):1363-72.

17. Yanofski J, Croarkin P. Choosing antidepressants for HIV and AIDS patients: insights on safety and side effects. Psychiatry (Edgmont). 2008;5(5):61-6.

18. Dubovsky AN, Arvikar S, Stern TA, Axelrod L. The neuropsychiatric complications of glucocorticoid use: Steroid psychosis revisited. Psychosomatics. 2012;53:103-15. 